\title{
Combined antiretroviral treatment and heterosexual transmission of HIV-1: cross sectional and prospective cohort study
}

\author{
Jorge Del Romero, clinical researcher, ${ }^{1}$ Jesús Castilla, consultant medical epidemiologist, ${ }^{2}$ Victoria Hernando, \\ epidemiologist, ${ }^{3}$ Carmen Rodríguez, research scientist, ${ }^{1}$ Soledad García, clinical researcher ${ }^{1}$
}

${ }^{1}$ Centro Sanitario Sandoval, Comunidad de Madrid, Spain ${ }^{2}$ Instituto de Salud Pública de Navarra, CIBER de Epidemiología y Salud Pública, Pamplona, Spain ${ }^{3}$ Centro Nacional de Epidemiología, Instituto de Salud Carlos III, CIBER de Epidemiología y Salud Pública, Madrid, Spain Correspondence to: J Del Romero jorgedelromero@gmail.com

Cite this as: BMJ 2010;340:c2205 doi:10.1136/bmj.c2205

\section{ABSTRACT}

Objective To estimate the risk and probability of heterosexual transmission of HIV-1 from infected people taking combined antiretroviral treatment.

Design Cross sectional and prospective cohort studies.

Setting HIV clinic in Madrid, Spain.

Participants Stable heterosexual couples with one partner with HIV-1 infection (index partner) and the other reporting this sexual relationship as the only risk exposure.

Main outcome measures HIV seroprevalence in non-index partners at enrolment and seroconversions in follow-up according to antiretroviral treatment taken by the index partner.

Results In 476 couples in which the index partner was not taking antiretroviral treatment, HIV seroprevalence at enrolment in non-index partners was 9.2\% $(n=44)$, whereas in 149 couples in which the index partner was taking combined antiretroviral therapy no partner was infected ( $P$ <0.001). During follow-up, the 341 serodiscordant couples in which the index partner was not taking antiretroviral treatment had about 11000 acts of intercourse without condoms, 50 natural pregnancies, and five HIV seroconversions (0.0004 per unprotected intercourse; $95 \%$ confidence interval 0.0001 to 0.0010 ); 294 of these couples always used condoms, accounting for about 42000 acts of intercourse, 136 risk exposures from condom failure, and one HIV seroconversion. The relative risk associated with condom use was 0.07 (0.01 to 0.58 ). In 144 couples the index partner was taking combined antiretroviral treatment; they accounted for over 7000 unprotected acts of intercourse and 47 natural pregnancies but no HIV seroconversion ( 0 to 0.0005 per unprotected intercourse).

Conclusions The heterosexual infectivity of HIV-1 in individuals taking effective antiretroviral treatment is low. Avoidance of unprotected intercourse and receipt of antiretroviral treatment by the infected partner in accordance with protocols are complementary measures to prevent HIV transmission.

\section{INTRODUCTION}

The probability of transmission of HIV-1 during each sexual contact varies considerably according to the type of sexual practice, presence of other sexually transmitted infections, stage of HIV disease, and plasma HIV RNA concentration in the infected partner. ${ }^{1-11}$

People with a stable heterosexual partner with HIV are exposed to the risk of infection each time their mucosa come into contact with their partner's genital fluids. Sexual abstinence is the only risk-free option but is not accepted by many partners who try to lead a normal sexual and reproductive life. ${ }^{12} 13$ The alternative is to use condoms in all sexual contacts, but systematic adherence is difficult for some couples, impedes natural conception, and does not completely eliminate the risk of transmission. ${ }^{14}$ Combined antiretroviral treatment can reduce HIV RNA in both the plasma ${ }^{15}$ and genital fluids of infected people, ${ }^{1617}$ and effective viral suppression has been associated with a significant reduction of infectivity. ${ }^{5-7}$ Some studies have associated the use of combined antiretroviral therapy with reduced sexual transmission of HIV, ${ }^{18-22}$ but none has established the probability of transmission in heterosexual relationships when the infected partner is receiving this treatment. ${ }^{23}$

We analysed stable heterosexual couples in which HIV-1 infection had been diagnosed in one partner (index partner) and the non-index partner reported sexual relationship with the index partner as the only risk exposure. We evaluated HIV seroprevalence in non-index partners at enrolment, as well as seroincidence and probability of transmission during successive visits, according to the couple's sexual risk exposures and antiretroviral treatment in the index partner.

\section{METHODS}

Setting and study population

This study began in 1989, at the same time as a specific programme for heterosexual HIV serodiscordant couples was launched in a clinic for HIV and sexually transmitted infections in Madrid, Spain. Each patient with a diagnosis of HIV infection was advised that his or her sexual partner should visit the clinic. The programme includes comprehensive medical consultation 
Table 1|Characteristics of couples enrolled during 1989-2008 according to treatment of index partner. Figures are numbers (percentages) of participants unless stated otherwise

\begin{tabular}{|c|c|c|c|c|}
\hline & \multirow[b]{2}{*}{ Total } & \multicolumn{3}{|c|}{ Antiretroviral treatment* } \\
\hline & & No treatment & $\begin{array}{l}\text { Combined } \\
\text { treatment }\end{array}$ & P value $†$ \\
\hline All & $648(100)$ & $476(100)$ & $149(100)$ & \\
\hline Male index case & $535(83)$ & $385(81)$ & $130(87)$ & 0.08 \\
\hline History of injecting drug use in index case & $494(76)$ & $370(78)$ & $106(71)$ & 0.1 \\
\hline \multicolumn{5}{|l|}{ Median (IQR) age (years): } \\
\hline Women & $29(25-35)$ & $28(24-33)$ & $34(30-39)$ & $<0.001$ \\
\hline Men & $32(28-37)$ & $30(27-35)$ & $38(33-40)$ & $<0.001$ \\
\hline Median (IQR) duration of relationship (years) & $3.1(1.0-7.6)$ & $2.9(0.9-7.0)$ & $4.2(1.4-9.5)$ & 0.004 \\
\hline Median (IQR) known duration of HIV infection (months) & $29(3-94)$ & $13(1-50)$ & $146(72-184)$ & $<0.001$ \\
\hline Began relationship after diagnosis of HIV in index case & $281(43)$ & $164(34)$ & $103(69)$ & $<0.001$ \\
\hline Unprotected penile-vaginal or penile-anal contact in past 6 months & $352(54)$ & $273(57)$ & $69(46)$ & 0.02 \\
\hline Unprotected penile-anal contact in past 6 months & $93(14)$ & $79(17)$ & $12(8.1)$ & 0.01 \\
\hline Previous pregnancy & $286(44)$ & $226(47)$ & $53(36)$ & 0.01 \\
\hline Current pregnancy & $42(6.5)$ & $32(6.7)$ & $8(5.4)$ & 0.7 \\
\hline Median (IQR) CD4 cell count $\times 10^{6} / \mathrm{l}$ in index partner $\ddagger$ & $500(295-700)$ & $514(291-716)$ & $475(300-660)$ & 0.4 \\
\hline Median (IQR) plasma HIV RNA copies per ml in index partner§ & 200 (ND-8876) & $8972(1069-41359)$ & ND & $<0.001$ \\
\hline Detectable plasma HIV RNA in index case & $143(54)$ & $111(93)$ & $30(21)$ & $<0.001$ \\
\hline History of AIDS defining condition & $107(17)$ & $43(9.0)$ & $57(38)$ & $<0.001$ \\
\hline Current sexually transmitted infection in either partner & $33(5.1)$ & $26(5.5)$ & $6(4.0)$ & 0.7 \\
\hline Current bacterial vaginosis or vaginal candidiasis in the woman & $100(15)$ & $72(15)$ & $16(11)$ & 0.2 \\
\hline Period 1997-2008 (combined treatment available) & $296(46)$ & $143(30)$ & 149 (100) & $<0.001$ \\
\hline HIV infection in non-index partner & $46(7.1)$ & $44(9.2)$ & 0 & $<0.001$ \\
\hline
\end{tabular}

$\mathrm{IQR}=$ interquartile range; $\mathrm{ND}=$ non-detectable plasma HIV RNA.

*Couples with index case taking monotherapy or dual therapy are not shown.

$\uparrow P$ values were obtained from Fisher's exact test or Wilcoxon's test.

$\ddagger$ Available for 573 patients.

§Available for 267 patients.

for both members of the couple, with check ups scheduled every six months. At each visit, the index partner undergoes clinical follow-up, the partner is recommended to undergo an HIV test, genitourinary infections (including sexually transmitted infections, bacterial vaginosis, and vaginal candidiasis) are ruled out, and women are offered an annual gynaecological examination. Access to free antiretroviral treatment is provided when the index partner meets current international guidelines. ${ }^{24}$ Couples are systematically advised against having unprotected sex. The staff responsible for this programme did not change throughout the study period.

\section{Design and variables}

With the informed consent of both partners, stable heterosexual couples treated in this programme were prospectively included in an observational study to quantify the risk of heterosexual HIV transmission according to sexual risk behaviours.

In a cross sectional analysis of HIV seroprevalence, we included couples recruited in 1989-2008, when the non-index partner came to the clinic for his or her first HIV test, who met the following criteria: ongoing sexual relationship during the past six months, the index partner had received a diagnosis of HIV with a well identified probable route of infection, and the nonindex partner had no previous HIV diagnosis and no known risk exposure other than the heterosexual relationship with the index partner. HIV prevalence in non-index partners was evaluated according to treatment of the index partner.

We included all heterosexual couples who were serodiscordant for HIV who returned for at least one follow-up visit in a prospective cohort analysis of HIV seroconversion. Follow-up began on the date of the first negative HIV test result within the programme, and the end point was the first HIV-1 positive test result. For those participants who failed to return for check ups for more than 24 months, whoserelationship ended, or whose non-index partner reported any risk exposure outside the relationship, data were censored at the last follow-up visit. For the remaining couples, follow-up was censored at the last check up before 31 December 2008.

We used a series of forms designed at the beginning of the study to collect epidemiological, clinical, and sexual behaviour information; these forms were subsequently modified to incorporate new laboratory tests and treatments. At all visits trained medical practitioners collected data. They asked non-index partners about the number of acts of sexual intercourse (vaginal and anal) during the previous six months (at the first visit) or since the previous visit (follow-up). For each type of practice, respondents reported the number of protected (with condom) and unprotected contacts using a semi-quantitative scale (never, less than half of the times, more than half of the times, and always); 


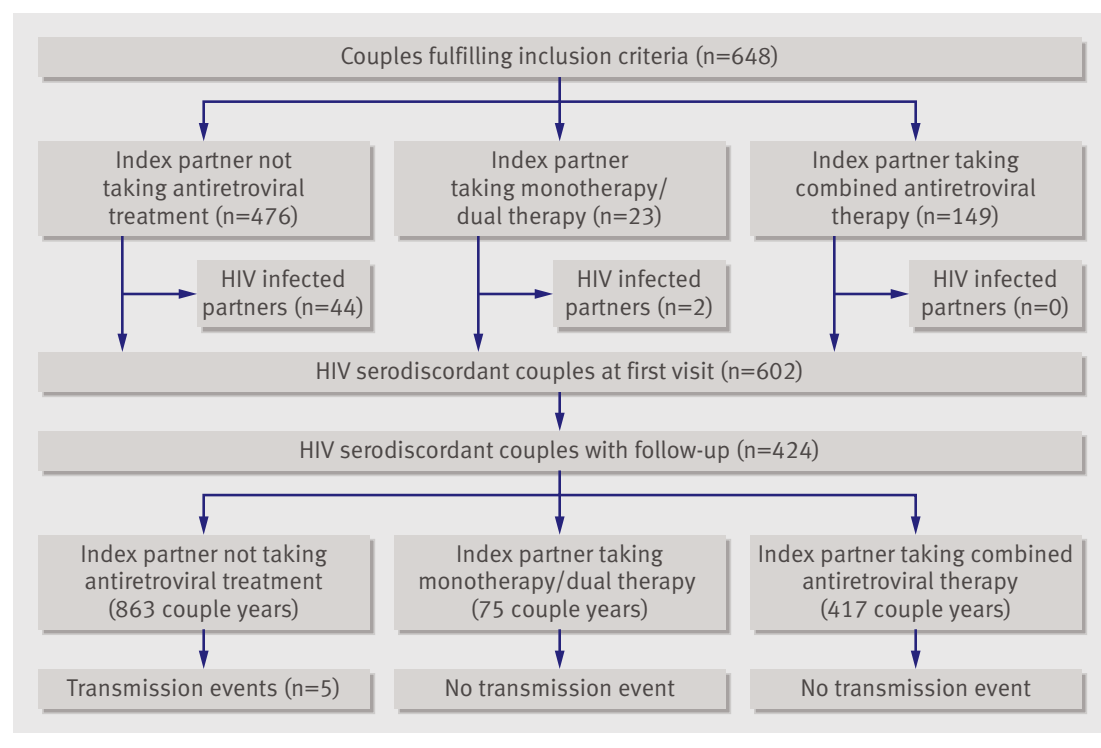

Study scheme of couples included in each analysis

to estimate frequencies we assigned the coefficients 0 , $0.33,0.67$, and 1 , respectively. Vaginal or anal intercourse without a condom was considered to be a "sexual risk practice." "Risky sexual exposures" included sexual risk practices and condom breakage or slippage during intercourse.

For each visit we categorised couples according to antiretroviral therapy of the index partner: not taking antiretroviral treatment, taking monotherapy/dual therapy, and taking combined therapy with at least three active drugs. Only treatments taken for at least three months were considered.

\section{Laboratory tests}

At baseline and every visit thereafter each partner provided a venous blood sample. Non-index partners were tested to determine serum antibodies to HIV-1/2, and reactive samples were confirmed by western blotting. Syphilis was routinely evaluated with a reaginic test and by Treponema pallidum passive particle agglutination assay or the fluorescent treponema antibody absorption test. Men were evaluated if they had symptoms of a sexually transmitted infection or if one had been diagnosed in their partner. Gynaecological examinations included screening for infections in cervical and vaginal exudates. CD4 count in the index partner was determined by flow cytometry and plasma HIV RNA by a branched DNA assay. The lower limit of detection was 500 copies per ml until 1999, and 50 copies per ml thereafter.

\section{Statistical analysis}

We estimated HIV seroprevalence at enrolment, the incidence rate of seroconversion by couple years of follow-up, and the probability of transmission per sexual risk exposure. We compared estimates for couples in which the index partner was taking combined antiretroviral therapy with those for couples in which the index partner was not receiving any antiretroviral treatment. In the follow-up analysis, we evaluated HIV seroconversions per couple years and per sexual risk exposures between two successive visits with respect to sexual behaviours, antiretroviral treatment, and characteristics of the couple reported for that specific period. Index partners who started or changed antiretroviral treatment between two successive visits were classified in the lower treatment category. Genitourinary infections and other circumstances detected at a visit were considered as covariates present during the whole time period since the previous visit.

We compared continuous variables with the Wilcoxon test, proportions with Fisher's exact test, and rates with exact methods. Confidence intervals for rates were calculated by assuming a Poisson distribution, and confidence intervals for risks by assuming a binomial distribution.

\section{RESULTS}

Prevalence of HIV infection at recruitment

Between 1989 and 2008, we recruited 648 heterosexual couples in which only one partner had previously received a diagnosis of $\mathrm{HIV}-1$ infection. The median length of the relationship was 3.1 years, and in 535 $(83 \%)$ couples the index partner was the man. Seroprevalence in the non-index partners of these couples was $11 \%(38 / 352)$ in $1989-96$ and $2.7 \%(8 / 296)$ in 1997 2008, when combined antiretroviral therapy became available $(\mathrm{P}<0.001)$. In 476 couples $(73 \%)$ the index partner was not taking antiretroviral treatment: 333 because this was before antiretrovirals became available, 99 who did not meet the criteria for treatment, 21 who were still under evaluation, and 23 who declined treatment. In 23 couples the index partner was taking monotherapy or dual therapy, and in 149 couples he or she was taking combined antiretroviral treatment.

Index partners who were taking combined antiretroviral treatment had a significantly longer known duration of HIV infection than those not taking any antiretroviral treatment and had more often begun the relationship with their partner after the diagnosis of HIV or AIDS defining diseases. In those in whom we could determine viral load, it was detectable in $93 \%$ $(111 / 120)$ of those who were not taking antiretroviral treatment compared with $21 \%(30 / 145)$ of those who were taking combined treatment $(\mathrm{P}<0.001)$. The proportion of those who had had unprotected sexual intercourse in the past six months was slightly lower in couples in which the index partner was taking combined antiretroviral treatment (273/476 (57\%) $v 69 / 149(46 \%), \mathrm{P}=0.019)$, as was the proportion of couples with previous pregnancies (226/476 (47\%) v 53/149 (36\%), $\mathrm{P}=0.011)$ (table 1).

The prevalence of HIV-1 infection was $9.2 \%$ (44/476) among partners of index partners who were not taking antiretroviral treatment and $8.7 \%(2 / 23)$ in partners of index partners taking monotherapy/dual therapy; no HIV infection was detected in partners of index partners taking combined therapy $(0 / 149 ; \mathrm{P}<0.001)$ (figure). The stratified analyses ruled out the main 
Table 2| Prevalence of HIV infection at first visit among heterosexual partners of index partner with HIV, according to selected characteristics of couple. Figures are numbers and percentages.

\begin{tabular}{|c|c|c|c|c|c|}
\hline \multirow[b]{2}{*}{ Population analysed } & \multicolumn{2}{|c|}{ No antiretroviral treatment } & \multicolumn{2}{|c|}{ Combined antiretroviral treatment } & \multirow[b]{2}{*}{$P$ value* } \\
\hline & Infected/analysed & HIV prevalence (\%) & Infected/analysed & HIV prevalence (\%) & \\
\hline All partners & $44 / 476$ & 9 & $0 / 149$ & 0 & $<0.001$ \\
\hline \multicolumn{6}{|l|}{ Sex of index partner: } \\
\hline Male & $38 / 385$ & 10 & $0 / 130$ & 0 & $<0.001$ \\
\hline Female & $6 / 85$ & 7 & $0 / 19$ & 0 & 0.6 \\
\hline \multicolumn{6}{|l|}{ Beginning of relationship: } \\
\hline After HIV diagnosis in index partner & $11 / 164$ & 7 & $0 / 103$ & 0 & 0.008 \\
\hline Before HIV diagnosis in index partner & $33 / 312$ & 11 & $0 / 46$ & 0 & 0.01 \\
\hline \multicolumn{6}{|l|}{ Time since beginning of relationship (years): } \\
\hline$<5$ & $27 / 305$ & 9 & $0 / 85$ & 0 & 0.001 \\
\hline$\geq 5$ & $14 / 171$ & 10 & $0 / 64$ & 0 & 0.005 \\
\hline \multicolumn{6}{|l|}{ Previous pregnancy in woman: } \\
\hline No & $16 / 254$ & 6 & $0 / 96$ & 0 & 0.008 \\
\hline Yes & $28 / 222$ & 13 & $0 / 53$ & 0 & 0.004 \\
\hline \multicolumn{6}{|l|}{ Plasma HIV RNA in index partner: } \\
\hline Not detectable & $0 / 9$ & 0 & $0 / 115$ & 0 & NA \\
\hline Detectable & $6 / 110$ & 6 & $0 / 30$ & 0 & 0.3 \\
\hline Not available & $38 / 357$ & 11 & $0 / 4$ & 0 & 1 \\
\hline \multicolumn{6}{|l|}{ CD4 cell count per $\times 10^{6} / /$ in index partner: } \\
\hline$<200$ & $11 / 58$ & 19 & $0 / 15$ & 0 & 0.1 \\
\hline$\geq 200$ & $18 / 352$ & 5 & $0 / 130$ & 0 & 0.005 \\
\hline Not available & $15 / 66$ & 23 & $0 / 4$ & 0 & 0.6 \\
\hline \multicolumn{6}{|l|}{ AIDS defining conditions in index partner: } \\
\hline No & $33 / 433$ & 8 & $0 / 92$ & 0 & 0.003 \\
\hline Yes & $11 / 43$ & 26 & $0 / 57$ & 0 & $<0.001$ \\
\hline \multicolumn{6}{|l|}{ Genitourinary infection in either partner: } \\
\hline No & $35 / 383$ & 9 & $0 / 129$ & 0 & $<0.001$ \\
\hline Yes & 9/93 & 10 & $0 / 20$ & 0 & 0.4 \\
\hline \multicolumn{6}{|l|}{ Period: } \\
\hline $1989-96$ & $36 / 334$ & 11 & $0 / 0$ & NA & NA \\
\hline $\begin{array}{l}\text { 1997-2008 (combined treatment } \\
\text { available) }\end{array}$ & $8 / 142$ & 6 & $0 / 149$ & 0 & 0.003 \\
\hline
\end{tabular}

potential confounding factors as an explanation of this association (table 2).

\section{HIV-1 transmission during follow-up}

We evaluated HIV-1 transmission in the 424 couples who were initially serodiscordant and who had at least one follow-up visit between 1989 and 2008 (figure). A total of 1355 couple years of follow-up were accrued, with about 95000 acts of intercourse, in which a condom was not used in about 20000 . During one or more follow-up periods, 266 couples $(63 \%)$ reported sexual risk exposures, and 88 had pregnancies by natural methods. About $20 \%$ of couples $(n=86)$ met criteria for censored follow-up (five HIV seroconversions, 23 deaths of a partner, 46 ended relationships, and 12 nonindex partners who had had sexual contacts with another person); 249 (59\%) did not return for check up in 24 months with no known cause; and $89(21 \%)$ were still in follow-up at the end of the study.

There were about 11000 sexual risk exposures in 863 couple years when the index partner was not taking antiretroviral treatment. Genitourinary infection was detected in 61 couples $(18 \%)$, and the median plasma HIV RNA concentration in index partners was 6402 copies per ml (interquartile range 500-42 916). There were 50 natural pregnancies and five HIV seroconversions, which represents an incidence of 0.6 per 100 couple years and a probability of transmission of 0.0004 per unprotected contact $(95 \%$ confidence interval, 0.0001 to 0.0010$)$. Of these couples, $294(86 \%)$ had always used condoms during intercourse between at least two successive visits, accruing 627 couple years of follow-up and about 42000 episodes of intercourse with condoms; there were 136 instances of condoms breaking or slipping during intercourse (three accidental exposures per 1000 sexual relations with condom). Only two of the susceptible partners took antiretroviral prophylaxis after these accidental exposures. Six of these events coincided with natural pregnancies and one with HIV seroconversion, which represents a probability of transmission of 0.000024 per contact with a condom $(0.000001$ to 0.000153$)$. The relative 
Table $3 \mid$ Characteristics of couples and events occurring during follow-up according to antiretroviral treatment of index partner

\begin{tabular}{|c|c|c|c|c|}
\hline & \multirow[b]{2}{*}{ All couples } & \multicolumn{3}{|c|}{ Antiretroviral treatment of index partner* } \\
\hline & & $\begin{array}{l}\text { Without } \\
\text { treatment }\end{array}$ & $\begin{array}{l}\text { Monotherapy/ } \\
\text { dual therapy }\end{array}$ & $\begin{array}{l}\text { Combined } \\
\text { treatment }\end{array}$ \\
\hline \multicolumn{5}{|l|}{ Couples in follow-up: } \\
\hline All & 424 & 341 & 47 & 144 \\
\hline Male index partner (\%) & $351(83)$ & $279(82)$ & $33(70)$ & $117(81)$ \\
\hline Couple years & 1355 & 863 & 75 & 417 \\
\hline Estimated No of coital acts & 95000 & 62000 & 5000 & 28000 \\
\hline \multicolumn{5}{|l|}{ Couples with sexual risk exposures during follow-upt: } \\
\hline Couples with unprotected sexual contacts (\%) & $266(63)$ & $187(55)$ & $24(51)$ & $101(70)$ \\
\hline Couples with unprotected penile-anal contacts & 26 & 13 & 4 & 11 \\
\hline Estimated No of risky sexual exposures & 20000 & 11000 & 1600 & 7400 \\
\hline No of condom failures during intercourse & 198 & 166 & 14 & 18 \\
\hline Women with bacterial vaginosis or vaginal candidiasis & 61 & 53 & 9 & 6 \\
\hline Sexually transmitted infection in either partnerł & 9 & 8 & 1 & 2 \\
\hline History of AIDS defining disease in index partner & 55 & 19 & 13 & 35 \\
\hline First year of relationship & 62 & 54 & 0 & 8 \\
\hline Index partner with primary infection & 3 & 3 & 0 & 0 \\
\hline Plasma HIV RNA (undetectable/tested (\%)) & $142 / 220(65)$ & 28/95 (29) & $6 / 14(43)$ & $112 / 122(92)$ \\
\hline Median (IQR) plasma HIV RNA copies per ml & ND (ND-6900) & $6402(500-42916)$ & 5367 (ND-16 770) & ND \\
\hline \multicolumn{5}{|l|}{ Seroconversions to HIV in follow-up: } \\
\hline No of seroconversions & 5 & 5 & 0 & 0 \\
\hline Percentage of couples $(95 \% \mathrm{Cl})$ & $1.2(0.4$ to 2.7$)$ & $1.5(0.5$ to 3.4$)$ & $0(0$ to 7.5$)$ & $0(0$ to 2.5$)$ \\
\hline Rate per 100 couple years $(95 \% \mathrm{Cl})$ & $0.4(0.1$ to 0.9$)$ & $0.6(0.2$ to 1.4$)$ & $0(0$ to 6.1$)$ & $0(0$ to 1.1$)$ \\
\hline Transmission per 1000 risk exposures $(95 \% \mathrm{Cl})$ & $0.2(0.1$ to 0.6$)$ & $0.4(0.1$ to 1.0$)$ & $0(0$ to 2.2$)$ & $0(0$ to 0.5$)$ \\
\hline \multicolumn{5}{|l|}{ Natural pregnancies during follow-up: } \\
\hline No of couples with any natural pregnancy & 88 & 48 & 4 & 39 \\
\hline Percentage $(95 \% \mathrm{Cl})$ & 21 (17 to 25$)$ & 14 (11 to 18$)$ & $9(2$ to 20$)$ & 27 (20 to 35$)$ \\
\hline No of natural pregnancies & 101 & 50 & 4 & 47 \\
\hline Rate per 100 couple years $(95 \% \mathrm{Cl})$ & $7.5(6.1$ to 9.0$)$ & $5.8(4.4$ to 7.6$)$ & $5.3(1.7$ to 13.8$)$ & $11.3(8.5$ to 14.8$)$ \\
\hline $\begin{array}{l}\text { ND=not detectable. } \\
{ }^{*} \text { Each couple could have different therapeutic options } \\
\text { †Includes penile-vaginal or penile-anal contacts without } \\
\text { †Condylomata acuminata (8) and urethritis (1). }\end{array}$ & $\begin{array}{l}\text { follow-up. } \\
\text { om and condom }\end{array}$ & ing or slipning du & ntercourse. & \\
\hline
\end{tabular}

risk of HIV transmission per intercourse with a condom compared with intercourse without a condom was 0.07 ( 0.01 to $0.58 ; \mathrm{P}=0.008$ ).

In 144 couples the index partner was taking combined antiretroviral treatment during 417 couple years, accruing over 7000 sexual risk exposures. Genitourinary infections were diagnosed in eight couples $(6 \%)$, and $92 \%(112 / 122)$ of index partners had undetectable plasma HIV RNA. During follow-up, 47 natural pregnancies occurred, but no seroconversion was detected (table 3). This provides an upper limit of the 95\% confidence interval for the probability of transmission of 0.0005 per unprotected relationship. There was, however, no significant difference between this probability and the probability of transmission when the index partner was not taking antiretroviral treatment $(\mathrm{P}=0.16)$.

\section{Characteristics of couples with HIV transmission}

HIV transmission was detected in 46 couples at the first visit and in five more couples during follow-up (table 4). Two of the 51 index partners were being treated with one antiretroviral drug, but none was receiving combined antiretroviral treatment. Two couples had not reported any unprotected intercourse and transmission was associated with a condom failing during intercourse; neither of these partners received antiretroviral prophylaxis after exposure. In four of the 51 couples with transmission, the diagnosis of HIV coincided with a natural pregnancy. In 12 couples $(29 \%)$ one or both partners had genitourinary infections, and 12 index partners had been diagnosed with AIDS defining conditions. The eight transmitter partners in whom plasma HIV RNA was measured had a detectable concentration, with a range of 362-214 400 copies per ml.

\section{DISCUSSION}

\section{Principal findings}

This study evaluated HIV-1 transmission in stable heterosexual serodiscordant couples, with prospective recording of risk exposures and other relevant factors. No index partner who was taking combined antiretroviral treatment transmitted the infection to his or her partner after 417 couple years of follow-up. This does not rule out the possibility of heterosexual transmission from people taking this treatment, but it allows us to establish, with less than $5 \%$ error, that the 
Table $4 \mid$ Characteristics of 51 couples at diagnosis of HIV infection in non-index partner. Figures are numbers (percentages) of participants or median (interquartile range)

\begin{tabular}{lc} 
All couples & No (\%) \\
Characteristics of index partner: & $44(86)$ \\
\hline Male & $41(80)$ \\
\hline Injecting drug user & $8(1-51)$ \\
\hline Median (IQR) time since HIV diagnosis (months) & $233(99-362)$ \\
\hline Median (IQR) last CD4 cell count $\times 10^{6} / \mathrm{I}(\mathrm{n}=36)$ & $54266(21264-163049)$ \\
\hline Median (IQR) last plasma HIV RNA copies per $\mathrm{ml}(\mathrm{n}=8)$ & $12(24)$ \\
\hline AIDS defining diseases & $2(4)$ \\
\hline Antiretroviral treatment: & $49(96)$ \\
\hline$\quad$ Monotherapy & \\
\hline No & $30(28-33)$ \\
\hline Characteristics of couple and relationship: & $26(22-32)$ \\
\hline Median (IQR) age (years): & $3.8(1.5-7.9)$ \\
\hline Men & $15(29)$ \\
\hline Women & $49(96)$ \\
\hline Median (IQR) time of relationship (years) & $15(29)$ \\
\hline Began relationship after HIV diagnosis in index partner & $2(4)$ \\
\hline Penile-vaginal contacts without condom & $4(8)$ \\
\hline Penile-anal contacts without condom & $12(24)$ \\
\hline Failure of condom during intercourse as sole risk exposure & $10(20)$ \\
\hline Pregnancy in woman & $46(90)$ \\
\hline Genitourinary infection in either partner & \\
\hline Transmission detected during combined antiretroviral treatment \\
period & \\
\hline Transmission detected at first visit & \\
\hline IQR=interquartile range. & \\
\hline
\end{tabular}

probability is less than 1 in 2000 risk exposures and that the rate of seroconversion is less than 1 in $91 \mathrm{cou}^{-}$ ple years. Moreover, we found no case of HIV transmission in the 149 couples at the first visit in which the index partner was taking combined antiretroviral therapy. Plasma HIV RNA was undetectable in most index partners taking combined antiretroviral treatment, which shows good control of viral replication. The viral load in genital secretions seems to fall together with the viral load in plasma after combined therapy, ${ }^{1617}$ and this would explain a reduction in infectivity. ${ }^{6}$

In the same programme, among attending couples in which the index partner was not taking combined antiretroviral therapy, we detected 46 HIV infections in the non-index partner at the first visit and five during follow-up. In the prospective analysis, condom use in all acts of intercourse reduced the risk of HIV transmission to the partner by $93 \%$ (95\% confidence interval $42 \%$ to $99 \%$ ) when the index partner was not taking antiretroviral treatment.

\section{Comparison with other studies}

The incidence of HIV infection found during followup in partners whose index partners were not taking combined antiretroviral treatment was lower than that reported in other studies. ${ }^{22}$ The probability of transmission from risk exposure in the follow-up was similar to or slightly lower than that described in studies in developed countries ${ }^{1-310}$ and much lower than in developing countries. ${ }^{4-68}$ The protective effect associated with condom use was within the range of that described in the literature. ${ }^{14}$

Although we did not find a single case of HIV transmission when the index partner was taking combined antiretroviral therapy, the existence of some risk could not be totally ruled out as the persistence of HIV in genital secretions or transient increases in viral load in patients taking combined antiretroviral treatment and long term suppression of the viral load in plasma have been described. ${ }^{162-28}$ Sexual transmission of HIV from an infected partner taking antiretroviral therapy with repeatedly undetectable plasma viral load has also been documented. ${ }^{29}$

\section{Strengths and limitations}

Participants were recruited within a programme offering preventive counselling, as well as diagnosis and treatment of genitourinary infections. During followup almost half of the couples discontinued their risk behaviour, and many others began to use condoms for most acts of intercourse. This would explain why the incidence rates during follow-up were lower than those that could be estimated from the cases of transmission detected at the first visit, before any influence of the intervention. Moreover, the infections detected at the first visit would include more vulnerable couples who rapidly become seroconcordant, whereas followup might over-sample less susceptible partners or less infective index partners. ${ }^{30}$

The couples in which the index partner was taking combined antiretroviral therapy were not completely comparable with those in which the index partner was not taking any antiretroviral treatment, given that antiretroviral therapy is prescribed for patients with worse clinical states,${ }^{24}$ who are therefore more infectious for their sexual partners. ${ }^{67}$

Although this is one of the studies with the largest number of couples and longest follow-up time of those conducted in developed countries, there was insufficient statistical power for some of the analyses, especially in situations with a low probability of transmission. Nevertheless, the number of exposures during follow-up was considerable, as shown by the 101 natural pregnancies that were registered.

Given the inclusion criteria, we can state with a high level of probability that the HIV infections detected in non-index partners were attributable to heterosexual transmission from the index partner. Caution advises us against generalising these results to casual partners or to homosexual relationships between men, given the more frequent circumstances favouring transmission, such as anal sex, presence of sexually transmitted infections, or early stage of infection. ${ }^{1-8}$

Analysis of self reported sexual behaviour data based on six month recall requires some caution. The quantification of exposures without condoms might also have been subject to some error as they were based on a categorical variable. In the context of a preventive intervention, the use of condoms might have been over-reported, leading to a higher estimate of 


\section{WHAT IS ALREADY KNOWN ON THIS TOPIC}

The probability of transmission of HIV-1 per sexual contact varies according to type of sexual practice, presence of sexually transmitted infections, stage of HIV disease, and plasma HIV RNA concentration in the infected partner

Combined antiretroviral therapy can reduce HIV RNA in both the plasma and genital fluids of infected people, and effective viral suppression has been associated with a significant reduction of infectivity

The infectivity of HIV-1 from individuals taking combined antiretroviral treatment to their heterosexual partners remains uncertain

\section{WHAT THIS STUDY ADDS}

No index partner taking combined antiretroviral treatment transmitted HIV infection to his/ her partner after numerous couple years of follow-up, sexual risk exposures, and natural pregnancies

Though there is a possibility of heterosexual transmission from people with HIV taking combined antiretroviral treatment, the risk of transmission is probably low

Avoidance of unprotected intercourse and receipt of combined antiretroviral treatment by the infected partner in accordance with protocols are complementary measures to prevent HIV transmission transmission is possible with condom failure during intercourse in serodiscordant couples. As to date no single measure has been able to guarantee the absence of the risk of HIV transmission during intercourse between serodiscordant heterosexual couples, the control of genitourinary infections, avoidance of unprotected intercourse, and antiretroviral treatment in the index partner in accordance with protocols should be considered as complementary measures.

Contributors: JDR, SG, and CR conceived the study. JC and VH contributed to the study design, statistical analysis, and data interpretation. JDR and JC wrote the manuscript and are guarantors. All authors contributed to the data interpretation and editing of the report, and all have seen and approved the final version.

Funding: This study was supported by a grant from FIPSE (foundation formed by the Spanish Ministry of Health and Consumer Affairs, Abbott Laboratories, Boehringer Ingelheim, Bristol Myers Squibb,

GlaxoSmithKline, Merck Sharp and Dohme, and Roche; No 24324/02 and by the Spanish Network for Research on AIDS (RIS), which is funded by the Instituto de Salud Carlos III. The authors affirm the independence of the researchers from the funders.

Competing interests: All authors have completed the Unified Competing Interest form at www.icmje.org/coi_disclosure.pdf (available on request from the corresponding author) and declare that they have no competing interests relevant to this work

Ethical approval: The study protocol was approved by the ethics

committee of the clinic

Data sharing: No additional data available. failures was underestimated; however, information was collected before the non-index partner's serological state was known, which reduces differential biases. Condom use might be more common in sexual relationships in which there are other concurrent risk factors - that is, higher risk practices, sexually transmitted infections, and high viral load in the index partner.

There were no cases of HIV transmission from index partners who were taking combined antiretroviral treatment, making it impossible for us to adjust multivariate models that control for potential confounders; consequently, we stratified by these factors in the retrospective analysis to rule out a confounding effect was causing a false association between antiretroviral treatment in the index partner and lower HIV seroprevalence in the couple.

Because this was a convenience cohort, with no incentives provided, the number of couples who dropped out without explanation was high. These dropouts are an important limitation in follow-up studies and can invalidate the results if they are related to HIV seroconversion in the non-index partner. Although the reason for not returning to the clinic could not be established in a large proportion of couples, we consider it was unlikely to be related with HIV seroconversion in the partner as the study was carried out in the most active and accessible HIV testing centre in the region.

\section{Conclusions and implications}

Although we cannot rule out the possibility of heterosexual transmission from people with HIV taking combined antiretroviral therapy, our results show that with these treatments the risk of transmission is probably low. Consistent condom use can have a considerable preventive impact in the population, although HIV
1 De Vincenzi I for the European Group on Heterosexual Transmission of HIV. A longitudinal study of human immunodeficiency virus transmission by heterosexual partners. $N$ Engl J Med 1994;331:341-6.

2 Saracco A, Musicco M, Nicolosi A, Angarano G, Arici C, Gavazzeni G, et al. Man-to-woman sexual transmission of HIV: longitudinal study of 343 steady partners of infected men. J Acquir Immune Defic Syndr 1993;6:497-502.

3 Padian NS, Shiboski SC, Glass SO, Vittinghoff E. Heterosexual transmission of human immunodeficiency virus (HIV) in northern California: results from a ten-year study. Am J Epidemiol 1997;146:350-7.

4 Wawer MJ, Gray RH, Sewankambo NK, Serwadda D, Li X Laeyendecker O, et al. Rates of HIV-1 transmission per coital act, by stage of HIV-1 infection, in Rakai, Uganda. J Infect Dis 2005;191:1403-9.

5 Gray RH, Wawer MJ, Brookmeyer R, Sewankambo NK, Serwadda D, act in monogamous, heterosexual, HIV-1-discordant couples in Rakai, Uganda. Lancet 2001;357:1149-53.

6 Quinn TC, Wawer MJ, Sewankambo N, Serwadda D, Li C, Wabwire-Mangen F, et al. Viral load and heterosexual transmission of human immunodeficiency virus type 1. N Engl J Med 2000;342:921-9.

7 Pedraza MA, del Romero J, Roldán F, García S, Ayerbe MC, Noriega AR, et al. Heterosexual transmission of HIV-1 is associated with high plasma viral load levels and a positive viral isolation in the infected partner. J Acquir Immune Defic Syndr 1999;21:120-5.

8 Fideli US, Allen SA, Musonda R, Trask S, Hahn BH, Weiss H, et al. Virologic and immunologic determinants of heterosexual transmission of human immunodeficiency virus type 1 in Africa. AIDS Res Hum Retroviruses 2001;17:901-10.

9 Powers KA, Poole C, Pettifor AE, Cohen MS. Rethinking the heterosexual infectivity of HIV-1: a systematic review and metaanalysis. Lancet Infect Dis 2008;8:553-63.

10 Downs AM, de Vincenzi I, European Study Group in Heterosexual Transmission of HIV. Probability of heterosexual transmission of HIV: relationship to the number of unprotected sexual contacts. J Acquir Immune Defic Syndr Hum Retrovirol 1996;11:388-95.

11 Boily MC, Baggaley RF, Wang L, Masse B, White RG, Hayes RJ, et al. Heterosexual risk of HIV-1 infection per sexual act: systematic review and meta-analysis of observational studies. Lancet Infect Dis 2009;9:118-29.

12 Centers for Disease Control and Prevention. Sexually transmitted diseases treatment guidelines, 2006. MMWR Recomm Rep 2006;55:1-94.

13 Coates TJ, Richter L, Caceres C. Behavioural strategies to reduce HIV transmission: how to make them work better. Lancet 2008;372:669-84. Wabwire-Mangen F, et al. Probability of HIV-1 transmission per coital 
14 Holmes KK, Levine R, Weaver M. Effectiveness of condoms in preventing sexually transmitted infections. Bull World Health Organ 2004;82:454-61.

15 Hammer SM, Squires KE, Hughes MD, Grimes JM, Demeter LM, Currier JS, et al. A controlled trial of two nucleoside analogues plus indinavir in persons with human immunodeficiency virus infection and CD4 cell counts of 200 per cubic millimeter or less. N Engl J Med 1997;337:725-33.

16 Sadiq ST, Taylor S, Kaye S, Bennett J, Johnstone R, Byrne P, et al. The effects of antiretroviral therapy on HIV-1 RNA loads in seminal plasma in HIV-positive patients with and without urethritis. AIDS 2002;16:219-25.

17 Vernazza PL, Troiani L, Flepp MJ, Cone RW, Schock J, Roth F, et al. Potent antiretroviral treatment of HIV-infection results in suppression of the seminal shedding of HIV. The Swiss HIV cohort study. AIDS 2000;14:117-21.

18 Porco TC, Martin JN, Page-Shafer KA, Cheng A, Charlebois E, Grant RM, et al. Decline in HIV infectivity following the introduction of highly active antiretroviral therapy. AIDS 2004;18:81-8.

19 Fang CT, Hsu HM, Twu SJ, Chen MY, Chang YY, Hwang JS, et al. Decreased HIV transmission after a policy of providing free access to highly active antiretroviral therapy in Taiwan. J Infect Dis 2004; 190:879-85.

20 Castilla J, del Romero J, Hernando V, Marincovich B, García S, Rodríguez C. Effectiveness of highly active antiretroviral therapy in reducing heterosexual transmission of HIV. J Acquir Immune Defic Syndr 2005;40:96-101.

21 Bunnell R, Ekwaru JP, Solberg P, Wamai N, Bikaako-Kajura W, Were W, et al. Changes in sexual behavior and risk of HIV transmission after antiretroviral therapy and prevention interventions in rural Uganda. AIDS 2006;20:85-92.
22 Attia S, Egger M, Müller M, Zwahlen M, Low N. Sexual transmission of HIV according to viral load and antiretroviral therapy: systematic review and meta-analysis. AIDS 2009;23:1397-404.

23 De Cock KM, Gilks CF, Lo YR, Guerma T. Can antiretroviral therapy eliminate HIV transmission? Lancet 2009;373:7-9.

24 Carpenter CC, Fischl MA, Hammer SM, Hirsch MS, Jacobsen DM, Katzenstein DA, et al. Antiretroviral therapy for HIV infection in 1998 Updated recommendations of the International AIDS Society-USA panel. JAMA 1998;280:78-86.

25 Neely MN, Benning L, Xu J, Strickler HD, Greenblatt RM, Minkoff H, et al. Cervical shedding of HIV-1 RNA among women with low levels of viremia while receiving highly active antiretroviral therapy. J Acquir Immune Defic Syndr 2007;44:38-42.

26 Di Mascio M, Markowitz M, Louie M, Hogan C, Hurley A, Chung C, et al. Viral blip dynamics during highly active antiretroviral therapy. J Virol 2003;77:12165-72.

27 Nettles RE, Kieffer TL, Kwon P, Monie D, Han Y, Parsons T, et al. Intermittent HIV-1 viremia (blips) and drug resistance in patients receiving HAART. JAMA 2005;293:817-29.

28 Van Sighem A, Zhang S, Reiss P, Gras L, van der Ende M, Kroon F, et al. Immunologic, virologic, and clinical consequences of episodes of transient viremia during suppressive combination antiretroviral therapy. J Acquir Immune Defic Syndr 2008;48:104-8.

29 Stürmer M, Doerr HW, Berger A, Gute P. Is transmission of HIV-1 in non-viraemic serodiscordant couples possible? Antivir Ther 2008;13:641-2.

30 Shiboski SC, Padian NS. Epidemiologic evidence for time variation in HIV infectivity. J Acquir Immune Defic Syndr Hum Retrovirol 1998;19:527-35.

Accepted: 1 April 2010 